\section{RMD Open}

Rheumatic \& Musculoskeletal Diseases

\title{
Long-term safety and efficacy of
} sarilumab plus methotrexate on disease activity, physical function and radiographic progression: 5 years of sarilumab plus methotrexate treatment

Mark C Genovese (D) , ${ }^{1}$ Désirée van der Heijde (D) ${ }^{2}$ Yong Lin, ${ }^{3}$ Gregory St John, ${ }^{4}$ Sheldon Wang, ${ }^{3}$ Hubert van Hoogstraten, ${ }^{3}$ Juan José Gómez-Reino, ${ }^{5}$ Alan Kivitz, ${ }^{6}$ José Antonio Maldonado-Cocco, ${ }^{7}$ Bruno Seriolo, ${ }^{8}$ Marina Stanislav, ${ }^{9}$ Gerd R Burmester (i) ${ }^{10}$

To cite: Genovese MC, van der Heijde D, Lin Y, et al. Long-term safety and efficacy of sarilumab plus methotrexate on disease activity, physical function and radiographic progression: 5 years of sarilumab plus methotrexate treatment. RMD Open 2019;5:e000887. doi:10.1136/ rmdopen-2018-000887

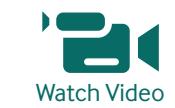
rmdopen.bmj.com

- Additional material is published online only. To view please visit the journal online (http://dx.doi.org/10.1136/ rmdopen-2018-000887).

MCG and DvdH contributed equally.

Received 20 December 2018 Revised 5 June 2019 Accepted 6 June 2019

Check for updates

\section{(c) Author(s) (or their} employer(s)) 2019. Re-use permitted under CC BY-NC. No commercial re-use. See rights and permissions. Published by BMJ.

For numbered affiliations see end of article.

\section{Correspondence to} Dr Mark C Genovese; Genovese@Stanford.edu

\section{ABSTRACT}

Objective In MOBILITY (NCT01061736), sarilumab significantly reduced disease activity, improved physical function and inhibited radiographic progression at week 52 versus placebo in patients with rheumatoid arthritis (RA) and an inadequate response to methotrexate. We report 5-year safety, efficacy and radiographic outcomes of sarilumab from NCT01061736 and the open-label extension (EXTEND; NCT01146652), in which patients received sarilumab $200 \mathrm{mg}$ every 2 weeks (q2w) + methotrexate.

Methods Patients $(n=1197)$ with moderately to severely active RA were initially randomised to placebo, sarilumab $150 \mathrm{mg}$ or sarilumab $200 \mathrm{mg}$ subcutaneously q2w plus weekly methotrexate for 52 weeks. Completers were eligible to enrol in the open-label extension and receive sarilumab $200 \mathrm{mg} \mathrm{q2w}+$ methotrexate.

Results Overall, 901 patients entered the open-label extension. The safety profile remained stable over 5-year follow-up and consistent with interleukin-6 receptor blockade. Absolute neutrophil count $<1000$ cells $/ \mathrm{mm}^{3}$ was observed but not associated with increased infection rate. Initial treatment with sarilumab $200 \mathrm{mg}$ + methotrexate was associated with reduced radiographic progression over 5 years versus sarilumab $150 \mathrm{mg}$ + methotrexate or placebo + methotrexate (mean \pm SE change from baseline in van der Heijde-modified Total Sharp Score: $1.46 \pm 0.27$, $2.35 \pm 0.28$ and $3.68 \pm 0.27$, respectively $(p<0.001$ for each sarilumab dose versus placebo)). Clinical efficacy was sustained through 5 years according to Disease Activity Score (28-joint count) using $C$ reactive protein, Clinical Disease Activity Index (CDAl) and Health Assessment Questionnaire-Disability Index. The number of patients achieving CDAl $\leq 2.8$ at 5 years was similar among initial randomisation groups (placebo, 76/398 (19\%); sarilumab $150 \mathrm{mg}, 68 / 400$ (17\%); sarilumab $200 \mathrm{mg}, 84 / 399$ (21\%)). Conclusion Clinical efficacy, including inhibition of radiographic progression, reduction in disease activity and improvement in physical function, was sustained with sarilumab + methotrexate over 5 years. Safety appeared stable over the 5-year period.

\section{Key messages}

What is already known about this subject?

- In the phase III MOBILITY trial (part B cohort 2; NCT01061736), sarilumab (150 and 200 mg every 2 weeks plus weekly methotrexate (MTX)) was shown to significantly reduce disease activity, improve physical function and inhibit radiographic progression at week 52 versus placebo (plus MTX) in adult patients with rheumatoid arthritis (RA) and an inadequate response to MTX.

What does this study add?

- In this open-label extension study, the safety profile of sarilumab remained stable over 5 -year follow-up and was consistent with interleukin 6 blockade, with most common adverse events being injection-site erythema, neutropenia, and upper respiratory tract infection.

- The clinical efficacy of sarilumab, including inhibition of radiographic progression, reduction in disease activity and improvement in physical function (according to DAS28-CRP, Clinical Disease Activity Index and Health Assessment Questionnaire-Disability Index), was sustained over 5 years. While initial treatment with either dose of sarilumab was associated with significantly better radiographic outcomes versus placebo over 5 years of follow-up, the best outcomes were observed in patients initially randomised to sarilumab $200 \mathrm{mg}$ in the double-blind study.

How might this impact on clinical practice or future developments?

- These results support the long-term use of sarilumab for adult patients with RA and an inadequate response to MTX.

\section{INTRODUCTION}

Rheumatoid arthritis (RA) is a chronic systemic inflammatory autoimmune disease 
characterised by persistent joint inflammation, which leads to bone and cartilage destruction, deformation and disability. ${ }^{1}$ In addition to progressive joint damage, RA is associated with a range of extra-articular manifestations, including cardiovascular disease, fatigue, pain and depression. ${ }^{2}$ The articular and systemic manifestations of RA are mediated, in part, by cytokines such as interleukin 6 (IL-6). ${ }^{34}$ IL-6 is a pleiotropic cytokine that plays a role in homeostasis, metabolism and regenerative processes. ${ }^{5} \mathrm{IL}-6$ levels increase locally in response to infection or injury, promoting proinflammatory activities. In autoimmune conditions, such as RA, persistently elevated IL-6 levels can contribute to chronic inflammation and disease progression. ${ }^{6}$

Sarilumab is a human monoclonal antibody that binds membrane-bound and soluble IL- 6 receptor- $\alpha$ to inhibit IL-6-mediated cis- and trans-signalling, and is approved for the treatment of adults with moderately to severely active RA. ${ }^{7}$ The safety and efficacy of sarilumab administered subcutaneously as monotherapy and in combination with conventional synthetic disease-modifying antirheumatic drugs (csDMARDs) have been demonstrated in active-comparator and placebo-controlled phase III trials in adults with RA. ${ }^{8-10}$

The phase III randomised controlled trial MOBILITY (part B cohort 2; NCT01061736) demonstrated superiority of subcutaneous sarilumab administered at doses of $200 \mathrm{mg}$ and $150 \mathrm{mg}$ every 2 weeks (q2w) plus methotrexate (MTX) compared with placebo plus MTX in reducing the signs and symptoms of RA, improving physical function and inhibiting progression of radiographic structural damage in patients with RA and an inadequate response to methotrexate (MTX-IR). ${ }^{8}$

Although the benefit-risk profile of sarilumab has been demonstrated in the short to medium term, the use of therapies in chronic disease necessitates longterm evaluation. Consequently, there is a need to evaluate safety and efficacy associated with long-term sarilumab use. ${ }^{11}$ This analysis assessed the safety and efficacy of sarilumab over 5 years of treatment in patients who completed the double-blind study and entered the open-label extension (EXTEND; NCT01146652).

\section{METHODS}

The multicentre, randomised, double-blind, placebocontrolled study has previously been described. ${ }^{8}$ The study was an operationally seamless phase II/III study. Part A was the phase II dose-ranging portion, and part B was the phase III portion of the study. Two cohorts of patients were enrolled in part B: those who were randomised before dose selection (cohort 1) and those who were randomised after dose selection (cohort 2). In part B cohort 2, MTX-IR adult patients with moderately to severely active RA were randomised (1:1:1) to placebo, sarilumab $150 \mathrm{mg}$ or sarilumab $200 \mathrm{mg}$ subcutaneously $\mathrm{q} 2 \mathrm{w}$ in combination with weekly MTX for 52 weeks. After 16 weeks, patients with $<20 \%$ improvement from baseline in either swollen joint count or tender joint count at two consecutive visits were offered rescue therapy with open-label sarilumab $200 \mathrm{mg}$ q2w for the remainder of the study. Patients who completed the study were eligible for enrolment in the open-label extension in which they received sarilumab $200 \mathrm{mg}$ q2w plus MTX. All patients continued to receive MTX background therapy, which could be reduced, discontinued or switched to an alternative approved non-biological DMARD for safety or tolerability reasons. Reasons for MTX discontinuation were not recorded. Patients were excluded from the open-label extension if they had adverse events (AEs) or other abnormalities that would adversely affect participation in the study, determined according to investigator judgement or protocol. The studies were conducted in accordance with Good Clinical Practice and with the principles stipulated in the Declaration of Helsinki; all protocols and patient information materials were approved by appropriate ethical review boards and all patients provided written informed consent.

Dose reduction to sarilumab $150 \mathrm{mg} \mathrm{q} 2 \mathrm{w}$ was permitted for patients who recovered following a laboratory event, which included absolute neutrophil count (ANC) $\geq 500-<1000$ cells $/ \mathrm{mm}^{3}$ (once ANC had returned to $\geq 1000$ cells $\left./ \mathrm{mm}^{3}\right)$, platelet count $\geq 50-<100 \times 10^{9}$ cells/L (once platelet count had returned to $\geq 100 \times 10^{9}$ cells/L), alanine aminotransferase (ALT) 3-5× the upper limit of normal (ULN; once ALT had returned to $<3 \mathrm{ULN}$ ) or per investigator judgement. Sarilumab was to be permanently discontinued in cases of significant laboratory abnormalities (ALT $>5 \times$ ULN or ALT $>3 \times$ ULN with concomitant total bilirubin $>2 \times$ ULN; neutrophil count $<500$ cells $/ \mathrm{mm}^{3}$ or neutrophil count $<1000$ cells $/ \mathrm{mm}^{3}$ with evidence of infection; platelet count $<50 \times 10^{9}$ cells $/$ L or platelet count $<100 \times 10^{9}$ cells $/ \mathrm{L}$ with evidence of bleeding), opportunistic infection, active tuberculosis, positive culture for non-tuberculosis mycobacteria, hypersensitivity or anaphylactic reactions, severe neurological disease, HIV positive status, acute renal failure, pregnancy, use of other biological agents or any AEs deemed by the investigator to jeopardise patient safety.

The last visit of the double-blind study was the first visit in the open-label extension. The primary objective of the open-label extension was to evaluate the long-term safety of sarilumab in patients with RA. The secondary objective was to assess the long-term efficacy of sarilumab. Safety and efficacy assessments and laboratory tests were conducted every 4 weeks up to week 12 , every 12 weeks up to week 96 and then at 24-week intervals. Additional laboratory testing, including haematology and liver function tests, were performed at week 2, week 6 and week 10 . AEs were collected throughout the study.

The safety population comprised all patients who received at least one dose of sarilumab. Safety assessments included incidences of treatment-emergent AEs, serious treatment-emergent AEs, adverse events of 
special interest (AESIs) and changes in specific laboratory parameters. Serious infections were defined as infections requiring hospitalisation and/or intravenous antibiotics. Major adverse cardiovascular events (MACE) were reviewed by an independent cardiovascular adjudication committee and suspected gastrointestinal perforations were confirmed by medical review. Leucopenia was included as an AESI to capture investigator-reported AEs of neutropenia. Thromboembolic events were not prespecified as an AESI in the study protocols but are reported here post hoc based on the Medical Dictionary for Regulatory Activities high-level group term 'Embolism and thrombosis'. Incidence rates by 6-month interval were analysed for serious adverse events (SAEs), serious infections, malignancies, MACE, injection-site reactions, ANC $<1000$ cells $/ \mathrm{mm}^{3}$, ALT $>3 \times$ ULN and platelet count $<100 \times 10^{9}$ cells/L. The exact method was used to calculate $95 \%$ CIs for proportions. Incidences of infection and serious infection were calculated by maximum neutropenia grade recorded at any time during the study. For infections that occurred within 12 weeks after an ANC assessment, incidence of infection or serious infection was calculated by the last ANC assessment before the onset of the infection.

Clinical efficacy assessments included Disease Activity Score (28 joints) using $\mathrm{C}$ reactive protein (DAS28-CRP) and proportion of patients achieving DAS28-CRP $<3.2$ and <2.6; Clinical Disease Activity Index (CDAI) and proportion of patients achieving CDAI $\leq 10$ (CDAI low disease activity) and $\leq 2.8$ (CDAI remission); and Health Assessment Questionnaire-Disability Index (HAQ-DI) and proportion of HAQ-DI responders (change from baseline of $\geq 0.22$ ). Efficacy results are presented as observed, including patients who received rescue medication with sarilumab $200 \mathrm{mg} \mathrm{q} 2 \mathrm{w}$ but excluding patients who discontinued study medication, without imputation for missing data. Responder rates are presented as a percentage of the intention-to-treat (ITT) population and as a percentage of the number of patients assessed (observed case approach). There was no confirmatory analysis for the efficacy variables. The baseline value for efficacy parameters was the original baseline from the double-blind study.

Radiographic progression was assessed by change from baseline in van der Heijde-modified Total Sharp Score (mTSS) recorded in four reading campaigns (one campaign in the double-blind phase and three campaigns in the open-label extension). In a reading campaign, independent readers were presented with a predefined set of images comprising radiographs of hands and feet from specific timepoints during the study. Readers were blinded to the chronological order, patient identity and treatment group. A post hoc integrated analysis was conducted to analyse radiographic progression based on all available campaign data. Repeated measurements by visit were analysed using a linear mixed-effects model with baseline mTSS, region and prior biological use as covariates and visit, treatment and the interaction of visit and treatment in the model and nested subjects in each campaign (campaign (reader) was a random effect as conducted previously $\left.{ }^{12}\right)$. Compound symmetry was chosen as a variance correlation structure. The change from baseline in mTSS at each timepoint was estimated by leastsquares means. The percentage of patients with no mTSS progression at the end of the open-label extension (change from baseline $\leq 0$ and $\leq 0.5$ ) was analysed in the last reading campaign. For this analysis, data collected after treatment discontinuation or starting rescue medication were used as observed. The linear extrapolation method was used to impute missing mTSS. Patients with still missing mTSS after the imputation step were considered as progressors. All analyses were performed using SAS version 9.2 or above (SAS Institute, Cary, North Carolina, USA).

\section{RESULTS}

\section{Patient characteristics and disposition}

Of the 1197 adult patients who entered the double-blind trial (placebo, $\mathrm{n}=398$; sarilumab $150 \mathrm{mg}, \mathrm{n}=400$; sarilumab $200 \mathrm{mg}, \mathrm{n}=399$ ), more patients in the placebo group $(\mathrm{n}=156)$ than in the sarilumab $150 \mathrm{mg}$ group $(\mathrm{n}=55)$ or sarilumab $200 \mathrm{mg}$ group $(\mathrm{n}=46)$ received rescue therapy. A total of 901 patients completed the double-blind trial and enrolled in the open-label extension: 307 from the placebo group, 300 from the sarilumab $150 \mathrm{mg}$ group and 294 from the sarilumab $200 \mathrm{mg}$ group. Two patients were not treated; therefore, 899 received sarilumab $200 \mathrm{mg}$ (online supplementary figure 1). During the open-label extension, discontinuation rates were similar across original treatment groups (figure 1). Overall in the double-blind study and the

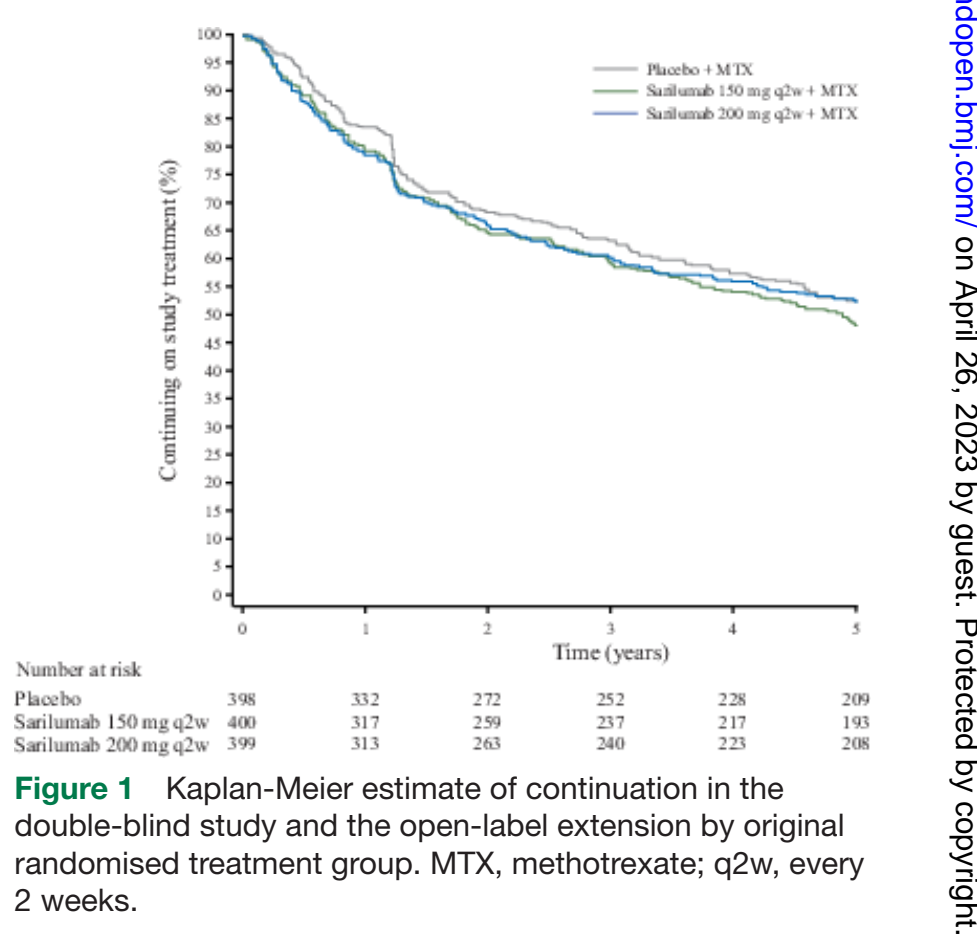


Table 1 Demographics and disease activity at entry into the double-blind study by original randomisation group for the double-blind $(n=1197)$ and open-label extension $(n=901)$ populations

\begin{tabular}{|c|c|c|c|c|c|c|}
\hline \multirow[b]{3}{*}{ Parameter at double-blind study baseline } & \multicolumn{3}{|c|}{ Double-blind study } & \multicolumn{3}{|c|}{ Open-label extension } \\
\hline & \multirow[b]{2}{*}{$\begin{array}{l}\text { Placebo + } \\
\text { MTX }(n=398)\end{array}$} & \multicolumn{2}{|c|}{ Sarilumab q2w + MTX } & \multirow[b]{2}{*}{$\begin{array}{l}\text { Placebo + } \\
\text { MTX }(n=307)\end{array}$} & \multicolumn{2}{|c|}{ Sarilumab q2w + MTX } \\
\hline & & $\begin{array}{l}150 \mathrm{mg} \\
(\mathrm{n}=400)\end{array}$ & $\begin{array}{l}200 \mathrm{mg} \\
(\mathrm{n}=399)\end{array}$ & & $\begin{array}{l}150 \mathrm{mg} \\
(\mathrm{n}=300)\end{array}$ & $\begin{array}{l}200 \mathrm{mg} \\
(\mathrm{n}=294)\end{array}$ \\
\hline Female, n (\%) & $321(81)$ & $319(80)$ & $337(84)$ & $246(80)$ & $241(80)$ & $246(84)$ \\
\hline Age, mean (SD), years & $50.9(11.2)$ & $50.1(11.9)$ & $50.8(11.8)$ & $50.8(10.7)$ & $50.3(11.8)$ & $50.2(11.6)$ \\
\hline Prior biological DMARD use, n (\%) & $86(22)$ & $87(22)$ & $84(21)$ & $71(23)$ & $75(25)$ & $64(22)$ \\
\hline Seropositive for RF, n (\%) & $336(84)$ & $345(87)^{\star}$ & $328(83)^{*}$ & $260(85)$ & $261(88) \dagger$ & $250(85) \dagger$ \\
\hline Seropositive for anti-CCP autoantibody, n (\%) & $340(85)$ & $359(90) \ddagger$ & $337(85) \ddagger$ & $264(86)$ & $273(91)$ & $255(87) \S$ \\
\hline Tender joint count (0-68), mean (SD) & $26.8(13.7)$ & $27.2(14.1)$ & $26.5(14.5)$ & $26.8(13.6)$ & $27.4(14.4)$ & $26.9(14.4)$ \\
\hline Swollen joint count (0-66), mean (SD) & $16.7(9.3)$ & $16.6(9.0)$ & $16.8(9.7)$ & $17.1(9.4)$ & $16.7(9.2)$ & $17.0(9.5)$ \\
\hline
\end{tabular}

*For RF, $\mathrm{n}=396$ for sarilumab $150 \mathrm{mg}$ and $\mathrm{n}=397$ for sarilumab $200 \mathrm{mg}$.

†For RF, $\mathrm{n}=298$ for sarilumab $150 \mathrm{mg}$ and $\mathrm{n}=293$ for sarilumab $200 \mathrm{mg}$.

‡For anti-CCP, $\mathrm{n}=398$ for sarilumab $150 \mathrm{mg}$ and $\mathrm{n}=293$ for sarilumab $200 \mathrm{mg}$.

$\S n=293$.

CCP, cyclic citrullinated peptide; CRP, C reactive protein; DAS28-CRP, Disease Activity Score (28 joints) using C reactive protein; DMARD, diseasemodifying antirheumatic drug; HAQ-DI, Health Assessment Questionnaire-Disability Index; MTX, methotrexate; q2w, every 2 weeks; RA, rheumatoid arthritis; RF, rheumatoid factor; SD, standard deviation.

open-label extension, discontinuations due to safety reasons were reported in 340 patients (28\%); discontinuations due to non-safety reasons were reported in 222 patients (19\%), including 52 patients (4\%) due to lack of efficacy and 29 (2\%) due to poor compliance with the protocol. At baseline of the double-blind study, demographics and disease characteristics of patients who enrolled in the open-label extension were similar both between treatment groups and to those of the total population who were randomised in the double-blind study (table 1). Demographics and patient characteristics were also similar across original randomisation groups at baseline of the open-label extension (online supplementary table 1 ).

\section{Adverse events}

Across the double-blind study and the open-label extension, mean exposure to either dose of sarilumab was 3.3 years (median exposure: 4.1 years), maximum exposure was 5.9 years, cumulative exposure was 3767 patient-years (PY), and 476 patients $(42 \%)$ were treated for $\geq 240$ weeks (approximately 5 years; online supplementary table 2). The exposure-adjusted incidence rates of AEs and SAEs were 137.7 and 9.1 per 100 PY, respectively, for patients receiving either dose of sarilumab (table 2). The most common AEs (with any dose of sarilumab) were injection-site erythema (incidence rate 13.5 per $100 \mathrm{PY}$ ), neutropenia (12.8 per $100 \mathrm{PY}$ ) and upper respiratory tract infection (7.6 per $100 \mathrm{PY}$; table 2). The most common AESIs were infections (incidence rate 55.1 per $100 \mathrm{PY}$ ), injection-site reactions (21.6 per $100 \mathrm{PY}$ ) and leucopenia (17.7 per $100 \mathrm{PY}$; online supplementary table 3 ). The incidence rate of AEs was generally stable over $>5$ years of treatment and there was no signal for an increased rate over time for any of the AEs (including SAEs and serious infections) when analysed by 6-month interval (online supplementary figure 2).

\section{Laboratory abnormalities}

Elevations of ALT to $>3 \times$ ULN occurred in 158 patients $(14 \%)$ receiving either dose of sarilumab and normalised on treatment in $84(53 \%)$ of these patients (online supplementary table 4 ). ANC $<1000$ cells $/ \mathrm{mm} 3$ occurred in 143 patients (13\%) receiving either dose of sarilumab and normalised on treatment in $104(73 \%)$ of these patients. Platelet counts $<100 \times 10^{9}$ cells /L were observed in 33 patients (3\%) receiving either dose of sarilumab and normalised on treatment in $20(61 \%)$ of these patients.

\section{Infections}

Serious infections occurred at a rate of 3.9 events per $100 \mathrm{PY}$ in patients treated with either dose of sarilumab (online supplementary table 3). The incidence of infections and serious infections was similar between patients with and without a recorded event of neutropenia at any time during the study (online supplementary table 5). Moreover, the incidence of infection was similar between patients with a lowest on-study ANC of $\geq 1500$ cells $/ \mathrm{mm}^{3}$-lower limit of normal (grade 1 neutropenia), $\geq 1000-<1500$ cells $/ \mathrm{mm}^{3}$ (grade 2) and $\geq 500-<1000$ cells $/ \mathrm{mm}^{3}$ (grade 3 ). Of the total 2109 infections observed, 1879 (49.1 events per 100 PY) 
Table 2 Investigator-reported treatment-emergent AEs in the double-blind study and the open-label extension combined

\begin{tabular}{|c|c|c|c|}
\hline \multirow[b]{2}{*}{$\mathrm{AE}$} & \multicolumn{3}{|l|}{$n_{E}\left(n_{E} / 100 P Y\right)$} \\
\hline & $\begin{array}{l}\text { Sarilumab } 150 \mathrm{mg} \\
\text { initial dose + MTX }\end{array}$ & $\begin{array}{l}\text { Sarilumab } 200 \mathrm{mg} \\
\text { initial dose }{ }^{\star}+\text { MTX }\end{array}$ & $\begin{array}{l}\text { Any sarilumab } \\
\text { dose†+ MTX }\end{array}$ \\
\hline \multicolumn{4}{|l|}{ Summarył } \\
\hline Any $\mathrm{AE}$ & $299(182.3)$ & $668(142.0)$ & 1041 (137.7) \\
\hline SAE & $36(10.3)$ & $183(9.9)$ & $313(9.1)$ \\
\hline AE leading to discontinuation & $56(16.1)$ & $172(8.4)$ & $318(8.4)$ \\
\hline AE leading to death & $2(0.6)$ & $7(0.3)$ & $16(0.4)$ \\
\hline \multicolumn{4}{|c|}{ AEs with incidence rate $\geq 5$ per $100 \mathrm{PY}$ in any dose group } \\
\hline Cumulative total $\mathrm{AE}$ observation period, $\mathrm{PY}$ & 355.5 & 2082.5 & 3826.0 \\
\hline Injection-site erythema & $77(21.7)$ & $267(12.8)$ & $518(13.5)$ \\
\hline Neutropenia & $64(18.0)$ & $235(11.3)$ & $491(12.8)$ \\
\hline Upper respiratory tract infection & $47(13.2)$ & $145(7.0)$ & $289(7.6)$ \\
\hline Accidental overdoseף & $30(8.4)$ & $137(6.6)$ & $220(5.8)$ \\
\hline Urinary tract infection & $24(6.8)$ & $124(6.0)$ & $213(5.6)$ \\
\hline ALT increased & $43(12.1)$ & $108(5.2)$ & $211(5.5)$ \\
\hline Viral upper respiratory tract infection & $32(9.0)$ & $84(4.0)$ & $172(4.5)$ \\
\hline Bronchitis & $19(5.3)$ & $104(5.0)$ & $173(4.5)$ \\
\hline Injection-site pruritus & $28(7.9)$ & $75(3.6)$ & $132(3.5)$ \\
\hline Influenza & $19(5.3)$ & $53(2.5)$ & $111(2.9)$ \\
\hline Headache & $22(6.2)$ & $41(2.0)$ & $83(2.2)$ \\
\hline
\end{tabular}

*Including placebo patients from the double-blind phase who switched to sarilumab $200 \mathrm{mg}$ in the open-label extension.

†Any dose includes exposure on all sarilumab doses.

\#lncidence rate $\left(n_{E} / 100 P Y\right)$ for summary is over time to first event.

§Incidence rate $\left(n_{E} / 100 \mathrm{PY}\right)$ is over cumulative total $A E$ observation period.

IAdministration of two or more doses of study drug during an interval $<11$ days.

$A E$, adverse event; ALT, alanine aminotransferase; MTX, methotrexate; $n_{E}$, number of events; $n_{E} / 100$ PY, number of events per 100 PY; PY, patient-years; SAE, serious adverse event.

occurred within 12 weeks after an ANC assessment. ANC values were normal at the last ANC assessment before infection for the majority of infections occurring within 12 weeks after an ANC assessment (1652/1879 (88\%); online supplementary table 6$)$. Similar results were observed for serious infection: ANC values were normal at the last ANC assessment before serious infection for $125 / 130(96 \%)$ serious infections occurring within 12 weeks of an ANC assessment. Herpes zoster infection was reported in 19 patients (1.6\%; 0.5 events per 100 $\mathrm{PY})$; all cases were non-disseminated.

\section{Other safety findings}

Malignancies occurred in 22 patients $(1.9 \%$; 0.6 events per $100 \mathrm{PY}$ ). The most common malignancies (occurring in $>1$ patient) were basal cell carcinoma $(n=4$; $0.4 \%)$, malignant melanoma $(\mathrm{n}=3 ; 0.3 \%)$ and breast cancer $(n=2 ; 0.2 \%)$. There were five confirmed cases of gastrointestinal perforation $(0.1$ events per $100 \mathrm{PY})$ : two upper and three lower (online supplementary table 3 ). Lipid elevations were reported in 149 patients treated with any dose of sarilumab (13.1\%; 5.6 events per $100 \mathrm{PY})$ and included increased levels of total cholesterol $(\mathrm{n}=15 ; 1.3 \%$; 0.5 events per $100 \mathrm{PY})$, low-density lipoprotein cholesterol ( $\mathrm{n}=12 ; 1 \% ; 0.4$ events per 100 $\mathrm{PY})$ and high-density lipoprotein cholesterol $(\mathrm{n}=2$; $0.2 \%$; $<0.1$ events per $100 \mathrm{PY})$. There were $13 \mathrm{MACE}$ ( $1.1 \%$; 0.3 events per $100 \mathrm{PY})$, which consisted of cardiovascular death $(n=5)$, myocardial infarction $(n=4)$ and stroke $(n=4)$. Thromboembolic events (as reported by the investigators and evaluated post hoc; not a prespecified AESI) occurred at a rate of 0.9 per $100 \mathrm{PY}$.

\section{Radiographic outcomes}

This integrated analysis incorporates radiographic data from four different reading campaigns that assessed data at years 5, 4, 3 and baseline $(\mathrm{n}=660$ patients; campaign 4), years 3,2 and baseline ( $\mathrm{n}=717$; campaign $3)$, years 2, 1 and baseline ( $\mathrm{n}=810$; campaign 2$)$ and year 1 , month 6 and baseline $(n=1158$; campaign 1$)$. At 5 years, mean (SE) changes from baseline in mTSS score were $1.46(0.27), 2.35(0.28)$ and $3.68(0.27)$ in the sarilumab $200 \mathrm{mg}$, sarilumab $150 \mathrm{mg}$ and placebo initial randomisation groups, respectively $(p<0.001$ for each sarilumab dose vs placebo). In the groups initially assigned to sarilumab 150 or $200 \mathrm{mg}$, mTSS progression was reduced compared with the group initially assigned to placebo throughout the open-label extension with 


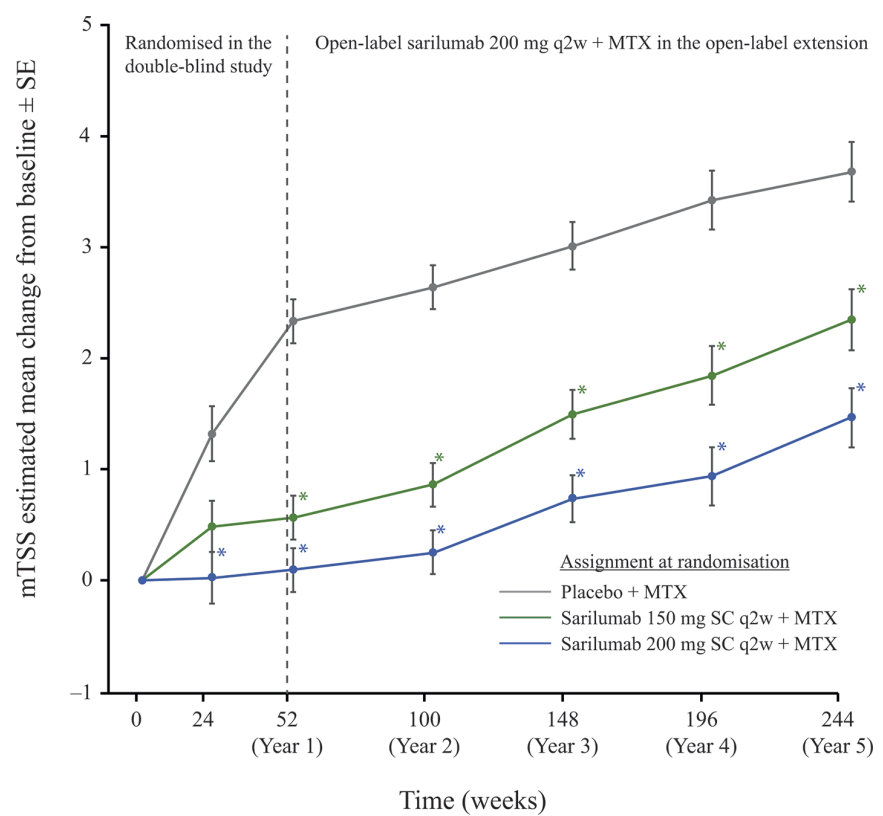

Figure 2 Estimated mean change from baseline in mTSS. *Nominal $p<0.001$ versus placebo. mTSS, van der Heijdemodified Total Sharp Score; MTX, methotrexate; q2w, every 2 weeks; SC, subcutaneously.

sarilumab $200 \mathrm{mg}$ (figure 2). The trajectory of mean mTSS progression in the group initially assigned to placebo showed a marked inflection at 1 year, coinciding with the switch to open-label sarilumab 200 $\mathrm{mg}$. Notably, the different trajectories of radiographic progression observed between the three treatment groups from year 1 were sustained over the succeeding years of follow-up; patients initially treated with sarilumab $200 \mathrm{mg}$ sustained a lower mean mTSS score at 5 years than patients initially treated with either sarilumab $150 \mathrm{mg}$ or placebo.

At 5 years, the proportion of patients with no mTSS progression (change from baseline $\leq 0$ ) was $47.1 \%$ $(107 / 221), 42.2 \%(94 / 223)$ and $37.2 \%(87 / 234)$ in the sarilumab $200 \mathrm{mg}$, sarilumab $150 \mathrm{mg}$ and placebo initial randomisation groups, respectively. Results were the same for mTSS non-progression defined as change from baseline $\leq 0.5$.

\section{Clinical efficacy}

Clinical efficacy according to DAS28-CRP, CDAI and HAQ-DI was sustained through 5 years of follow-up. At year 5, mean (SE) DAS28-CRP scores were similar among initial randomisation groups: sarilumab 200 $\mathrm{mg}, 2.21$ (0.07); sarilumab $150 \mathrm{mg}, 2.36(0.08)$ and placebo, $2.43(0.09)$ (figure 3A), having decreased by $62 \%$ with sarilumab $200 \mathrm{mg}$ from $5.97(0.04)$ at baseline (based on 208/399 patients with data at year 5). Likewise, the proportions of patients achieving DAS28-CRP $<2.6$ and $<3.2$ were similar among initial randomisation groups after 1 year of open-label sarilumab treatment in the extension study (ie, at 2 years of follow-up) and remained generally similar thereafter to 5 years of
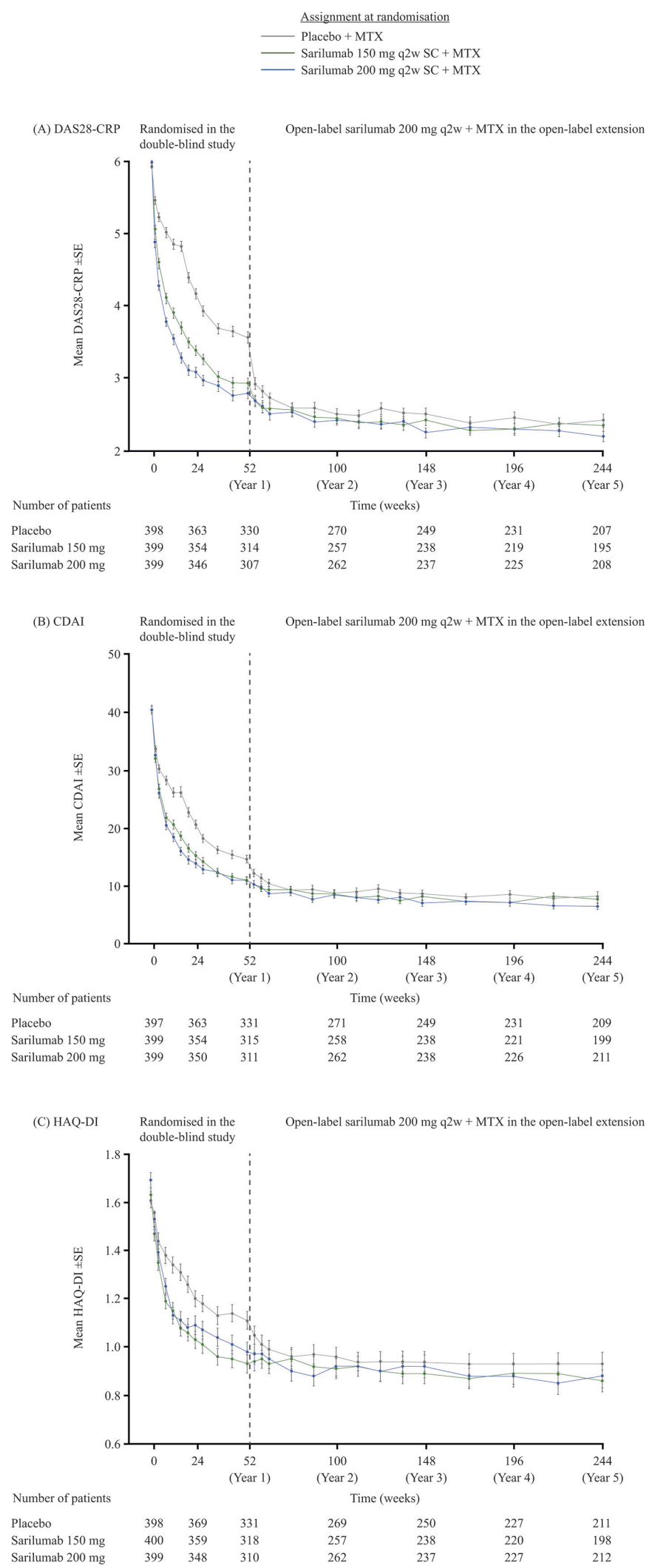

Figure 3 Mean clinical efficacy scores over time, as observed, without imputation for missing patients: $(A)$ DAS28-CRP, (B) CDAl and (C) HAQ-DI. CDAI, Clinical Disease Activity Index; DAS28-CRP, Disease Activity Score (28 joints) using $C$ reactive protein; HAQ-DI, Health Assessment Questionnaire-Disability Index; MTX, methotrexate; q2w, every 2 weeks; SC, subcutaneously. 


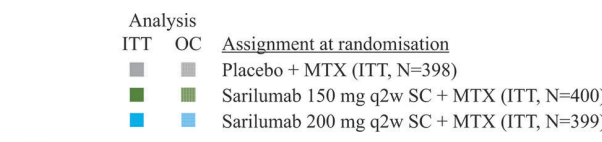

(A)

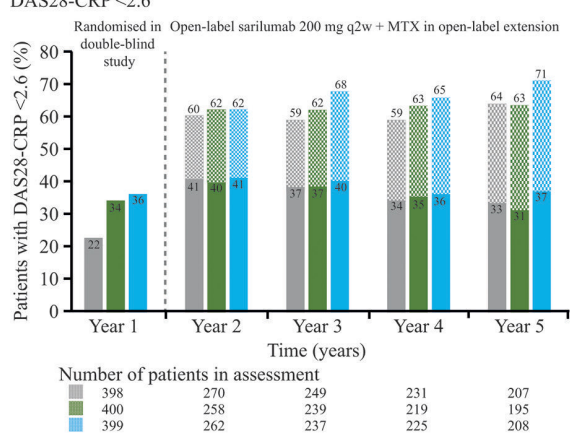

(C)

$\mathrm{CDAI} \leq 2.8$

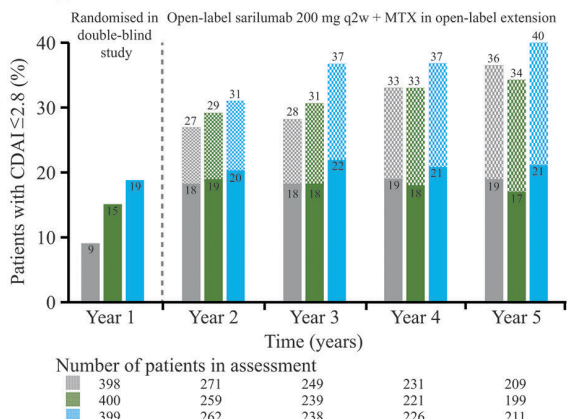

(E)

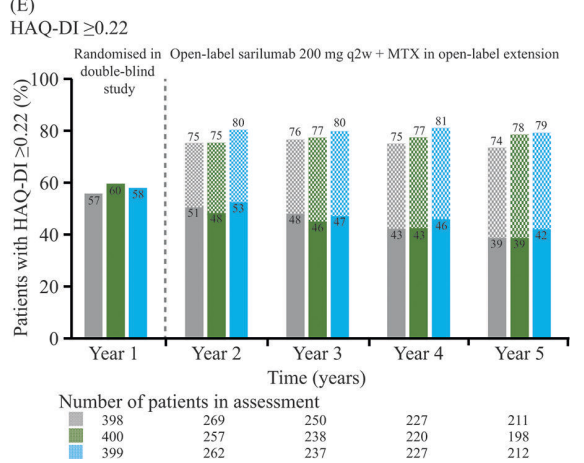

(B)

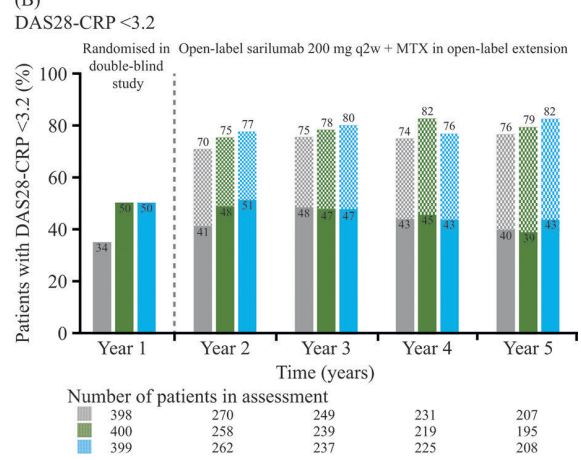

(D)

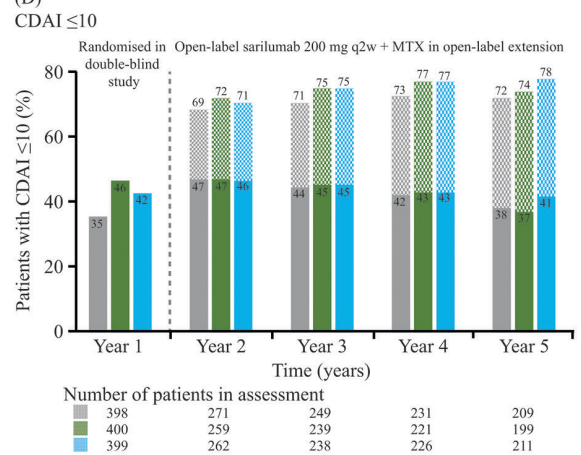

(F)

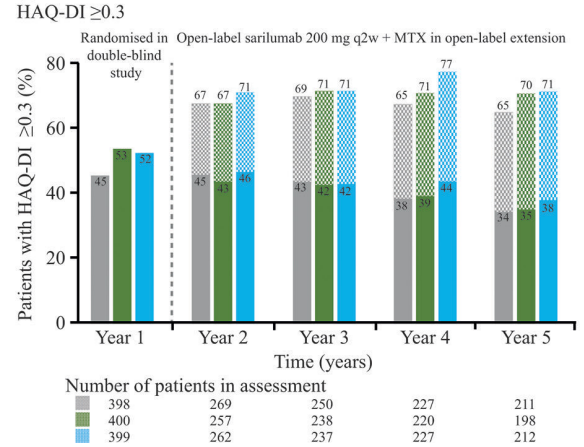

Figure 4 Proportions of patients at each year achieving: (A) DAS28-CRP $<2.6$, (B) DAS28-CRP <3.2, (C) CDAI $\leq 2.8$, (D) CDAI $\leq 10$, (E) HAQ-DI $\geq 0.22$ and (F) HAQ-DI $\geq 0.30$. In the ITT analysis, the denominator for percentages at all timepoints is the ITT population. In OC analysis, the denominator for percentages is the number of patients in the assessment at that timepoint. CDAI, Clinical Disease Activity Index; DAS28-CRP, Disease Activity Score (28 joints) using C reactive protein; HAQ-DI, Health Assessment Questionnaire-Disability Index; ITT, intention to treat; MTX, methotrexate; OC, observed cases; q2w, every 2 weeks; SC, subcutaneously.

follow-up (figure 4A,B). Results for CDAI were similar to those for DAS28-CRP. At year 5, mean (SE) CDAI was $6.56(0.49), 7.76(0.63)$ and $8.38(0.68)$ in the sarilumab $200 \mathrm{mg}$, sarilumab $150 \mathrm{mg}$ and placebo initial randomisation groups, respectively (figure 3B), having decreased by $83 \%$ with sarilumab $200 \mathrm{mg}$ from 40.4 (0.62) at baseline (based on 211/399 patients with data at year 5 ). The proportions of patients achieving CDAI $\leq 2.8$ and $\leq 10$ were similar among initial randomisation groups after 1 year of open-label sarilumab treatment in the extension study (ie, at 2 years of follow-up) and remained generally similar thereafter through 5 years of follow-up (figure 4C,D). Results for physical function, assessed by HAQ-DI, were similar to those observed for DAS28-CRP and CDAI; mean (SE) HAQ-DI scores at 5 years were $0.88(0.049), 0.86(0.046)$ and $0.93(0.049)$ in the sarilumab $200 \mathrm{mg}$, sarilumab $150 \mathrm{mg}$ and placebo initial randomisation groups, respectively (figure 3C), having decreased by $47 \%$ with sarilumab $200 \mathrm{mg}$ from 1.69 (0.03) at baseline (based on 211/399 patients with data at year 5$)$. The proportions of patients achieving HAQ-DI $\geq 0.22$ and $\geq 0.30$ were similar among initial 
randomisation groups after 1 year of open-label sarilumab treatment in the extension study (ie, at 2 years of follow-up) and remained generally similar thereafter through 5 years of follow-up (figure $4 \mathrm{E}, \mathrm{F}$ ).

\section{Dose reduction and MTX discontinuation}

Dose reduction from sarilumab 200 to $150 \mathrm{mg}$ occurred in 177 patients (20\% of 899 patients treated with sarilumab $200 \mathrm{mg}$ ). The most common reasons for dose reduction were ANC decrease $(n=108,61 \%$ of patients who reduced their dose) and ALT elevation $(n=44,25 \%)$. Following temporary discontinuation, sarilumab was reinitiated at the reduced dose of $150 \mathrm{mg}$ once ANC levels returned to $\geq 1000$ cells $/ \mathrm{mm}^{3}$ or ALT levels returned to $<3 \times$ ULN. ANC reductions and ALT elevations started to improve within 1 month of dose reduction and improvements continued thereafter (online supplementary table 7). Clinical efficacy was sustained after dose reduction (online supplementary figure 3 ).

In total, 92 patients discontinued MTX during the open-label extension: 10 patients switched to an alternative approved csDMARD and 82 patients permanently discontinued MTX and received sarilumab monotherapy. Clinical efficacy was sustained in those patients remaining in the study after MTX discontinuation.

\section{DISCUSSION}

The safety and efficacy of sarilumab $200 \mathrm{mg}$ and $150 \mathrm{mg}$ plus MTX were previously demonstrated in MTX-IR patients with moderately to severely active RA in the 52-week double-blind study. ${ }^{8}$ Results from this 5-year analysis of the open-label extension study confirm the long-term safety and efficacy of sarilumab in this group of patients.

The safety profile of sarilumab was stable over 5 years of follow-up and observations were generally consistent, not only with those in the randomised portion of the study but also with other studies in the sarilumab RA clinical programme. ${ }^{8-1013}$ In addition, the safety findings are consistent with the anticipated safety profile of IL-6 blockade. ${ }^{14}$ No new safety signals emerged and the most common AEs were injection-site erythema, neutropenia, upper respiratory tract infection, urinary tract infections and increased ALT. Occurrence of injection-site reactions was common (21.6 events per $100 \mathrm{PY}$ ) but consistent with long-term data for subcutaneous tocilizumab (26.1 events per $100 \mathrm{PY}$ ), another IL-6 receptor antagonist. ${ }^{15}$ Neutropenia was also common with sarilumab treatment, which is consistent with the phase III clinical trials of sarilumab and with this class of therapy. ${ }^{8-10} 14$ However, despite neutropenia occurring at a rate of 12.8 events per $100 \mathrm{PY}$ at any sarilumab dose, the infection rate reported in these patients was low, which suggests that neutropenia related to sarilumab treatment is not associated with an increased risk of infection. Furthermore, the last ANC recorded before onset of infection or serious infection was normal in the majority of cases. These results are consistent with a post hoc analysis of three sarilumab phase III trials and patients entering the open-label extensions of two of these trials. ${ }^{16}$ The post hoc analysis also found that the majority of patients in the randomised controlled trials and open-label extensions who temporarily discontinued treatment with sarilumab due to low ANC were able to continue or reinitiate sarilumab with no apparent clinically meaningful impact on long-term efficacy or safety. ${ }^{16}$ Published data suggest that patients with RA are at a twofold higher risk of herpes zoster infection than individuals without RA, ${ }^{17}$ but the reported rate was low in the open-label extension $(0.5$ events per $100 \mathrm{PY})$ and comparable to the general population $(0.3-0.5$ events per $100 \mathrm{PY}) .{ }^{18}$ While the absolute incidence of AEs was lower in the patients initially randomised to sarilumab $150 \mathrm{mg}$ than those receiving the $200 \mathrm{mg}$ dose, the exposure-adjusted incidence rates were higher. This result should be interpreted with caution, due to the limited duration of exposure in the sarilumab $150 \mathrm{mg}$ group and the relatively small number of events. Furthermore, the absolute incidence of AEs in the double-blind phase was lower in patients randomised to sarilumab $150 \mathrm{mg}$ than those randomised to sarilumab $200 \mathrm{mg} .{ }^{8}$ No increases in rates of AEs or SAEs were observed with increasing exposure duration and the incidence rates of AEs and laboratory abnormalities generally remained stable when analysed over time by 6 -month interval. Furthermore, the incidences of injection-site reactions and ALT $>3 \times$ ULN declined over time.

It has previously been reported that patients with RA are at an increased risk of gastrointestinal perforation, which is a rare but SAE. ${ }^{19}$ Although gastrointestinal perforations were reported infrequently over long-term sarilumab use in the extension study (two upper and three lower gastrointestinal perforations), the protocol exclusion of patients with a history of diverticulitis-another recognised risk factor for gastrointestinal perforation ${ }^{19}$ may have mitigated against the risk of gastrointestinal perforation in this population. Investigation of potential gastrointestinal perforation should take into consideration the $\mathrm{C}$ reactive protein-lowering effect of IL-6 signalling inhibition.

Evidence suggests that patients with active, untreated RA have reduced total, low-density lipoprotein and highdensity lipoprotein cholesterol levels, despite a $50 \%$ higher cardiovascular risk than the general population. ${ }^{21} 22$ This effect is known as the lipid paradox. Although increases in low-density lipoprotein cholesterol were observed in this open-label extension, MACE were reported infrequently. This supports previous findings with IL-6 receptor inhibitors suggesting that increases in serum lipids associated with effective RA immunosuppression do not increase cardiovascular event risk. ${ }^{23}$ Other analyses across phase III clinical trials of sarilumab observed that sarilumab treatment was associated with an increase in lipid levels compared with placebo and adalimumab; however, levels of lipoprotein (a) (a cardiovascular risk marker) were reduced and lipid elevations were largely associated with a reduction in inflammatory markers (serum amyloid $\mathrm{A}$ and $\mathrm{C}$ reactive protein). ${ }^{24}{ }^{25}$ These results suggest that inhibition of IL-6 signalling does not affect cardiovascular risk, despite the effects on lipid levels. 
Preventing joint damage progression is an important therapeutic goal in RA and joint damage can progress in patients despite clinically quiescent disease. ${ }^{26}$ Initial treatment with either dose of sarilumab was associated with significantly better radiographic outcomes versus placebo over 5 years of follow-up, with the best outcomes observed in patients initially randomised to sarilumab $200 \mathrm{mg}$ in the double-blind study. These treatment differences persisted through year 5 in the open-label extension, at which time all patients were receiving sarilumab $200 \mathrm{mg}$. This suggests that the excess structural damage observed in patients who had received placebo or sarilumab $150 \mathrm{mg}$ during the double-blind study likely occurred during that first year of study treatment. These results are consistent with other studies that have demonstrated improvements in long-term outcomes with early intensive treatment in patients with RA. ${ }^{27-31}$ Furthermore, these results support an analysis demonstrating suppression of circulating biomarkers of bone resorption and synovial damage with sarilumab plus MTX versus placebo plus MTX in MTX-IR patients. ${ }^{32}$

Clinical efficacy, according to DAS28-CRP, CDAI and HAQ-DI, was sustained through 5 years of follow-up. Similar changes from baseline in DAS28-CRP, CDAI and HAQ-DI were observed regardless of the initial randomisation group in the randomised portion of the study. Improvements in the proportions of patients achieving DAS28-CRP $<3.2$ and $<2.6$ and CDAI $\leq 10$ and $\leq 2.8$ continued over time. These results are consistent withand build on-the 2 year observations. ${ }^{13}$

Among the main limitations associated with long-term extension studies is enrichment of the cohort for patients who respond well to treatment and tolerate any AEs they encounter. As a result, the measurement of continuous variables can be influenced by the progressively smaller number of patients who remain in the study. To help minimise this bias, the absolute numbers of patients are presented at each relevant timepoint in addition to percentage response rates. Furthermore, ITT analyses are presented alongside completer analyses for dichotomous variables. ${ }^{11}$

In conclusion, the durable efficacy of sarilumab was demonstrated through 5 years of follow-up in an openlabel extension study, and reductions in disease activity, inhibition of radiographic progression and improvements in physical function were observed. No new safety signals were identified, and the safety profile remained stable and consistent with IL-6 blockade.

\footnotetext{
Author affiliations

${ }^{1}$ Stanford University Medical Center, Palo Alto, California, USA

${ }^{2}$ Leiden University Medical Centre, Leiden, The Netherlands

${ }^{3}$ Sanofi Genzyme, Bridgewater, New Jersey, USA

${ }^{4}$ Regeneron Pharmaceuticals Inc, Tarrytown, New York, USA

${ }^{5}$ Complejo Hospitalario Universitario de Santiago de Compostela, Santiago de Compostela, Spain

${ }^{6}$ Altoona Center for Clinical Research, Duncansville, Pennsylvania, USA ${ }^{7}$ School of Medicine, University of Buenos Aires, Buenos Aires, Argentina

${ }^{8}$ Department of Internal Medicine, University of Genova, Genova, Italy

${ }^{9}$ Research Rheumatology Institute n. a. V.A. Nasonova, Moscow, Russia
}

${ }^{10}$ Department of Rheumatology and Clinical Immunology, Charité - University Medicine Berlin, Berlin, Germany

Acknowledgements The authors thank the patients and their families, as well as the investigators and other study staff involved in the studies. The authors would also acknowledge Chunfu Qiu for providing statistical support for safetyrelated data. These data were previously presented in part at the 21st Asia Pacific League of Associations for Rheumatology Congress (APLAR) in conjunction with the Australian Rheumatology Association, 8-11 April 2019, Brisbane, Queensland, Australia: International Journal of Rheumatic Diseases 2019; 22 (Suppl 3): 206-207 (Abstract 2-100).

Contributors MCG, DvdH and HvH contributed to the design of the study; MCG, AK, JAM-C and MS contributed to data acquisition; YL contributed to data review and addressed data-related queries; and all authors contributed to data analysis and interpretation. All authors were involved in revising the manuscript critically for important intellectual content and approved the final version to be published.

Funding This study was supported by Sanofi and Regeneron Pharmaceuticals, Inc., which funded medical writing support by Natalie Roberts, PhD, Adelphi Communications.

Competing interests MCG has received research grants or consulting fees from R-Pharm, Roche/Genentech and Sanofi Genzyme. DvdH has received consulting fees from AbbVie, Amgen, Astellas, AstraZeneca, BMS, Boehringer Ingelheim, Celgene, Daiichi, Eli Lilly, Galapagos, Gilead, GlaxoSmithKline, Janssen, Merck, Novartis, Pfizer, Regeneron, Roche, Sanofi, Takeda and UCB. YL, SW and HvH are employees of Sanofi Genzyme and may hold stock and/or stock options in the company. GSJ is an employee of Regeneron and may hold stock and/or stock options in the company. JJG-R has received research support and/or consulting fees from Biogen, Gilead, Eli Lilly, Merck Sharp \& Dohme, Pfizer and Roche. AK has received consulting fees and/or participated in speakers' bureaus for AbbVie, Pfizer, Genentech, UCB, Sanofi/Regeneron, Celgene, Horizon and Merck. JAM-C has received consulting fees and/or participated in speakers' bureaus for Pfizer, Merck Sharp \& Dohme, Sanofi Aventis, Novartis, Bristol-Myers Squibb, Roche, Boehringer Ingelheim, Schering-Plough, Abbott, UCB, Eli Lilly and Gilead. MS has received consulting fees from $\mathrm{R}$-Pharm. GRB has received research support and/or consulting fees from AbbVie, Lilly, Merck Sharp \& Dohme, Pfizer, Roche, Sanofi and UCB. BS has nothing to disclose.

Patient consent for publication All patients provided written informed consent.

Ethics approval The MOBILITY and EXTEND protocols were approved by the appropriate ethics committees/institutional review boards.

Provenance and peer review Not commissioned; externally peer reviewed.

Data availability statement Qualified researchers may request access to patient level data and related study documents including clinical study report, study protocol with any amendments, blank case report form, statistical analysis plan, and dataset specifications. Patient level data will be anonymised and study documents will be redacted to protect the privacy of trial participants. Further details on Sanofi's data sharing criteria, eligible studies, and process for requesting access can be found at: https://www.clinicalstudydatarequest.com

Open access This is an open access article distributed in accordance with the Creative Commons Attribution Non Commercial (CC BY-NC 4.0) license, which permits others to distribute, remix, adapt, build upon this work non-commercially, and license their derivative works on different terms, provided the original work is properly cited, appropriate credit is given, any changes made indicated, and the use is non-commercial. See: http://creativecommons.org/licenses/by-nc/4.0/.

\section{ORCID iDs}

Mark C Genovese http://orcid.org/0000-0001-5294-4503

Désirée van der Heijde http://orcid.org/0000-0002-5781-158X

Gerd R Burmester http://orcid.org/0000-0001-7518-1131

\section{REFERENCES}

1 Picerno V, Ferro F, Adinolfi A, et al. One year in review: the pathogenesis of rheumatoid arthritis. Clin Exp Rheumatol 2015;33:551-8.

2 Choy EHS, Calabrese LH. Neuroendocrine and neurophysiological effects of interleukin 6 in rheumatoid arthritis. Rheumatology 2018;57:1885-95.

3 Firestein GS, McInnes IB. Immunopathogenesis of rheumatoid arthritis. Immunity 2017;46:183-96.

4 Schett G, Gravallese E. Bone erosion in rheumatoid arthritis: mechanisms, diagnosis and treatment. Nat Rev Rheumatol 2012;8:656-64. 
5 Schett G. Physiological effects of modulating the interleukin- 6 axis. Rheumatology 2018;57(suppl 2):ii43-50.

6 Tanaka T, Kishimoto T. Targeting interleukin-6: all the way to treat autoimmune and inflammatory diseases. Int J Biol Sci 2012;8:1227-36.

7 Kevzara Prescribing Information, 2017. Available: https://wwwaccessdatafdagov/drugsatfda_docs/ label/2017/761037s000lblpdf

8 Genovese MC, Fleischmann R, Kivitz AJ, et al. Sarilumab plus methotrexate in patients with active rheumatoid arthritis and inadequate response to methotrexate: results of a phase III study. Arthritis Rheumatol 2015;67:1424-37.

9 Fleischmann R, van Adelsberg J, Lin Y, et al. Sarilumab and nonbiologic disease-modifying antirheumatic drugs in patients with active rheumatoid arthritis and inadequate response or intolerance to tumor necrosis factor inhibitors. Arthritis Rheumatol 2017;69:277-90.

10 Burmester GR, Lin Y, Patel R, et al. Efficacy and safety of sarilumab monotherapy versus adalimumab monotherapy for the treatment of patients with active rheumatoid arthritis (MONARCH): a randomised, double-blind, parallel-group phase III trial. Ann Rheum Dis 2017;76:840-7.

11 Buch MH, Silva-Fernandez L, Carmona L, et al. Development of EULAR recommendations for the reporting of clinical trial extension studies in rheumatology. Ann Rheum Dis 2015;74:963-9.

12 Landewé R, Østergaard M, Keystone EC, et al. Analysis of integrated radiographic data from two long-term, open-label extension studies of adalimumab for the treatment of rheumatoid arthritis. Arthritis Care Res 2015:67:180-6.

13 Genovese MC, van Adelsberg J, Fan C, et al. Two years of sarilumab in patients with rheumatoid arthritis and an inadequate response to MTX: safety, efficacy and radiographic outcomes. Rheumatology 2018;57:1423-31

14 Kim GW, Lee NR, Pi RH, et al. IL-6 inhibitors for treatment of rheumatoid arthritis: past, present, and future. Arch Pharm Res 2015;38:575-84.

15 Burmester GR, Rubbert-Roth A, Cantagrel A, et al. Efficacy and safety of subcutaneous tocilizumab versus intravenous tocilizumab in combination with traditional DMARDs in patients with RA at week 97 (SUMMACTA). Ann Rheum Dis 2016;75:68-74.

16 Curtis JR, St. John G, Pannucci M, et al. Reductions in Absolute Neutrophil Count (ANC) with Sarilumab Resulting in Dose Delays or Dose Decreases: Effects on Efficacy and Safety [abstract]. Arthritis Rheumatol. 2018; 70 (suppl 10). https://acrabstracts.org/abstract/ reductions-in-absolute-neutrophil-count-anc-with-sarilumabresulting-in-dose-delays-or-dose-decreases-effects-on-efficacyand-safety/. Accessed November 22, 2018.

17 Smitten AL, Choi HK, Hochberg MC, et al. The risk of herpes zoster in patients with rheumatoid arthritis in the United States and the United Kingdom. Arthritis Care Res 2007:57:1431-8.

18 Kawai K, Gebremeskel BG, Acosta CJ. Systematic review of incidence and complications of herpes zoster: towards a global perspective. BMJ Open 2014:4:e004833.

19 Curtis JR, Lanas A, John A, et al. Factors associated with gastrointestinal perforation in a cohort of patients with rheumatoid arthritis. Arthritis Care Res 2012:64:1819-28.

20 Xie F, Yun H, Bernatsky S, et al. Brief report: risk of gastrointestinal perforation among rheumatoid arthritis patients receiving tofacitinib, tocilizumab, or other biologic treatments. Arthritis Rheumatol 2016:68:2612-7.

21 Peters MJL, Symmons DPM, McCarey D, et al. EULAR evidencebased recommendations for cardiovascular risk management in patients with rheumatoid arthritis and other forms of inflammatory arthritis. Ann Rheum Dis 2010;69:325-31.

22 Aviña-Zubieta JA, Choi HK, Sadatsafavi M, et al. Risk of cardiovascular mortality in patients with rheumatoid arthritis: a meta-analysis of observational studies. Arthritis Rheum 2008;59:1690-7.

23 Giollo A, Bissell L-A, Buch MH. Cardiovascular outcomes of patients with rheumatoid arthritis prescribed disease modifying anti-rheumatic drugs: a review. Expert Opin Drug Saf 2018;17:697-708.

24 Charles-Schoeman C, St John G, Leher H, et al. The relationship between lipid profile changes and inflammation across the phase 3 sarilumab rheumatoid arthritis $(\mathrm{RA})$ developmental program [abstract].Arthritis Rheumatol . 2018; 70 (suppl 10). Available: https://acrabstracts.org/abstract/the-relationship-between-lipidprofile-changes-and-inflammation-across-the-phase-3-sarilumabrheumatoid-arthritis-ra-developmental-program/ [Accessed November 22, 2018].

25 Gabay C, Msihid J, Paccard C, et al. Sarilumab significantly suppresses circulating biomarkers of bone resorption and cardiovascular risk compared with adalimumab: biomarker analysis from the phase 3 monarch study. Ann Rheum Dis 2017;76:570.

26 Steunebrink LMM, Versteeg LGA, Vonkeman HE, et al. Radiographic progression in early rheumatoid arthritis patients following initial combination versus step-up treat-to-target therapy in daily clinical practice: results from the DREAM registry. BMC Rheumatol 2018;2.

27 Lard LR, Visser H, Speyer I, et al. Early versus delayed treatment in patients with recent-onset rheumatoid arthritis: comparison of two cohorts who received different treatment strategies. The American Journal of Medicine 2001;111:446-51.

28 Nell VPK, Machold KP, Eberl G, et al. Benefit of very early referral and very early therapy with disease-modifying anti-rheumatic drugs in patients with early rheumatoid arthritis. Rheumatology 2004;43:906-14.

29 Quinn MA, Emery P. Window of opportunity in early rheumatoid arthritis: possibility of altering the disease process with early intervention. Clin Exp Rheumatol 2003;21:S154-7.

30 Steunebrink LMM, Versteeg GA, Vonkeman HE, et al. Initial combination therapy versus step-up therapy in treatment to the target of remission in daily clinical practice in early rheumatoid arthritis patients: results from the DREAM registry. Arthritis Res Ther 2016;18:60

31 Steunebrink LMM, Vonkeman HE, ten Klooster PM, et al. Recently diagnosed rheumatoid arthritis patients benefit from a treat-totarget strategy: results from the DREAM registry. Clin Rheumatol 2016;35:609-15.

32 Boyapati A, Msihid J, Fiore S, et al. Sarilumab plus methotrexate suppresses circulating biomarkers of bone resorption and synovia damage in patients with rheumatoid arthritis and inadequate response to methotrexate: a biomarker study of mobility. Arthritis Res Ther 2016;18:225. 